\title{
Coping em Jovens frente à Expectativa de Inserção Ocupacional e Indicadores de Depressão ${ }^{1}$
}

\author{
Jorge Artur Peçanha de Miranda Coelho² \\ Francisco José Batista de Albuquerque \\ Cíntia Ribeiro Martins \\ Henrique Barboza D’Albuquerque \\ Maria Tereza de Souza Neves \\ Universidade Federal da Paraíba
}

\begin{abstract}
RESUMO - O objetivo do presente trabalho consistiu em investigar o papel do pensamento construtivo e da estratégia de coping frente à expectativa de inserção ocupacional e suas conseqüências em relação a indicadores de depressão em uma população não clínica de estudantes. Participaram deste estudo 413 estudantes, sendo 35,7\% do terceiro ano do ensino médio e $24,4 \%$ do ensino superior da cidade de Areia (área rural), e 14,4\% do terceiro ano do ensino médio e 25,4\% do ensino superior da cidade de João Pessoa (área urbana). De acordo com os resultados, os jovens que utilizam os recursos de coping, tais como coping comportamental e emocional (dimensões do pensamento construtivo) e coping direto (dimensão das estratégias de coping), são os que melhor enfrentam as pressões da transição da escola/universidade para o mercado de trabalho, com menores níveis de depressão e melhores expectativas de inserção ocupacional.
\end{abstract}

Palavras-chave: inserção ocupacional; coping; depressão; jovens.

\section{Coping in Young Adults Facing Expectation of Finding a Job and Depression Indicators}

\begin{abstract}
The purpose of the present research was to investigate the role of constructive thinking and the coping strategy of facing the expectation of job insertion, and their consequences regarding depression indicators in a non-clinical student population. Four hundred and thirteen students participated in this study: $35.7 \%$ of them were in the last year of high school and $24.4 \%$ were university students from the city of Areia (rural area), and $14.4 \%$ were in the last year of high school and $25.4 \%$ were university students from the city of João Pessoa (urban area). According to the results, students who use coping resources such as behavioral and emotional coping (constructive thinking dimensions) and direct coping (coping strategies dimension) are those ones who best face transition pressures from school/university to the workplace with lower levels of depression and better expectations of job insertion.
\end{abstract}

Keywords: job insertion; coping; depression; young adults.

Ter uma ocupação remunerada e a sua falta são aspectos salientes no pensamento dos jovens. Três em cada quatro jovens brasileiros estão trabalhando ou tentando trabalhar. $\mathrm{O}$ trabalho é um dos temas de maior interesse dos jovens, em virtude de estar associado a conceitos do tipo necessidade, crescimento, independência e auto-realização, os quais fazem parte das suas maiores preocupações. A dificuldade encontrada para a inserção no mercado de trabalho, juntamente com o tema da violência, são percebidos como os principais componentes negativos de sua condição de jovens (Criterium Assessoria, 2004).

No Brasil, de acordo com Organização Internacional do Trabalho (OIT, 2001), as taxas de desemprego total e juvenil cresceram acentuadamente a partir de 1989. Contribuíram

1 O presente artigo é baseado em parte da Dissertação de Mestrado do primeiro autor, orientada pelo segundo autor e apresentada em 2005 no Programa de Pós-Graduação em Psicologia Social da UFPB. Apoio financeiro: $\mathrm{CNPq}$ e bolsa de mestrado da CAPES.

2 Endereço: Universidade Federal da Paraíba, CCHLA - Departamento de Psicologia, Núcleo de Estudos sobre Desenvolvimento Rural e Avaliação de Programas Sociais - NEDRAPS. João Pessoa, PB. CEP 58059-900.E-mail: jorgearturpmc@gmail.com. para isso a inserção da "economia brasileira no mercado internacional, de maneira mais competitiva; o processo de reestruturação econômica e de privatizações; as mudanças tecnológicas e organizacionais que, por sua vez, têm modificado a estrutura ocupacional e os perfis de competências" (p. 9). Segundo essa organização, o desemprego juvenil cresceu mais que o desemprego total, aproximadamente o dobro.

Sarriera, Schwarez e Câmara (1996a) salientam a importância que a ocupação tem sobre a saúde dos jovens e ressaltam que as atribuições psicossociais e econômicas do trabalho se apresentam como uma "constante preocupação na vida dos jovens e cumprem as funções de consolidar sua identidade, suprir as necessidades econômicas, desenvolver suas relações sociais, fornecer a prestação de serviços à comunidade e assegurar uma fonte de renda" (p. 74). Quando se comparam jovens empregados e desempregados, os desempregados têm um menor nível de bem-estar psicológico, menor auto-estima, maiores níveis de depressão e ansiedade, e menor grau de satisfação com a vida (Luque \& Estramiana, 2000).

Diante dessa constatação, além de levar em conta que no Brasil são escassos os estudos acerca do modo como os 
jovens lidam com os estressores da situação de transição da escola/universidade para o mercado de trabalho, o presente estudo objetivou investigar o pensamento construtivo e as estratégias de coping, como explicadores das expectativas de inserção ocupacional, e a tendência à depressão, em uma amostra não clínica de jovens do ambiente urbano e rural.

A Psicologia Social tem se concentrado nos estudos voltados para o ambiente urbano, deixando de lado o ambiente rural como fonte de preocupação; são poucas as pesquisas dedicadas a esse ambiente. No presente estudo, buscou-se focalizar tanto o ambiente urbano quanto o rural, por duas razões principais. Primeiro, por se pensar que, no Brasil, um posto de trabalho é tão importante no ambiente urbano quanto no ambiente rural. A importância do trabalho no ambiente rural se deve tanto ao fato de ser mais barato em termos de investimento, quanto por manter as pessoas nos seus lugares de origem, propiciando um desenvolvimento sustentável e minimizando os efeitos perversos do êxodo e, conseqüentemente, o crescimento desordenado das grandes cidades (Albuquerque, 2002). Segundo, o corpo de conhecimentos existente na Psicologia Social pode contribuir para a avaliação de programas de políticas públicas direcionadas a jovens, visando à promoção da saúde mental, do bem-estar social e da melhor qualidade de vida.

\section{Coping e Indicadores de Depressão}

Os fatores estressantes ameaçam o bem-estar psicológico, pois parecem exceder a capacidade das pessoas em lidar com o estresse. São exemplos de eventos estressantes: a perda de emprego, morte do cônjuge, enchentes, incêndios, entre outros. Mas o estresse também pode ser experimentado em função dos eventos do dia-a-dia, das pressões da luta cotidiana (Smith \& Mackie, 1997). A forma como os jovens enfrentam esses eventos da vida diária tem implicação na sua saúde mental e física, principalmente no tocante à depressão (Brissette, Scheier \& Caver, 2002; Caver \& Scheir, 1994). Pode-se definir a depressão como sendo um sentimento de profundo desânimo com o futuro, insatisfação com a vida ou isolamento dos outros; ausência de energia para fazer as coisas ou até mesmo para levantar da cama; incapacidade de concentração, de comer e de dormir normalmente; o pensamento de se não seria melhor estar morto (Myers, 1998).

No âmbito da Psicologia Social, o construto coping está relacionado ao modo como as pessoas enfrentam dificuldades ou circunstâncias estressantes. Coping consiste na capacidade que o indivíduo tem para pensar e agir da melhor forma possível, frente a situações de estresse. O coping constitui um importante aspecto do estresse e é uma das principais ferramentas para o seu manejo (Hudek-Knežecić \& Kardum, 1996), além de ser usualmente considerado como sendo o mediador entre saúde mental e física (Hudek-Knežecić \& Kardum, 2000; Ryan-Wenger, 1992).

Existem duas abordagens teóricas básicas para definir coping, a situacional e a estrutural. A abordagem estrutural trata dos estilos de coping, que têm sido mais relacionados a características de personalidade, enquanto a abordagem situacional diz respeito às estratégias de coping, que se referem a cognições e a ações tomadas no curso de um episódio estressor. Embora os estilos possam influenciar a extensão das estratégias, eles são fenômenos distintos e têm diferentes origens teóricas (Hudek-Knežecić \& Kardum, 2000; Ryan-Wenger, 1992).

O pensamento construtivo, que pode ser considerado como um estilo de coping, é compreendido como sendo a habilidade para resolver problemas, sem incorrer em um custo elevado de estresse e outras conseqüências negativas. Fundamenta-se em pensamentos producentes e contraproducentes (Epstein \& Meier, 1989). Uma pessoa com elevado índice global de pensamento construtivo pode ser considerada uma pessoa flexível, de fácil adaptação às situações, que não interpreta críticas e divergências com outras pessoas como sendo um insulto pessoal. Além disso, é otimista quando as situações permitem, sem ser ingênua (otimismo ingênuo), e apresenta uma orientação para a solução de problemas, ao invés de uma imposição de seus pontos de vista.

Avaliando as subescalas da medida de pensamento construtivo, denominadas de estilos de pensamento, proposta por Epstein e Meier (1989), pode-se afirmar que uma pessoa que apresenta fortemente o coping emocional (uma das seis subescalas) é capaz de enfrentar situações difíceis sem sofrer estresse em demasia. Já a subescala coping comportamental mede o quanto o indivíduo pensa de forma que promova ações efetivas. As pessoas que apresentam este estilo de pensamento em maior grau tendem a pensar de forma simplificada e maniqueísta, enquanto o estilo de pensamento categórico leva a ações rápidas e decisivas. O pensamento supersticioso, por sua vez, diz respeito às crenças das pessoas em relação a fenômenos que são cientificamente questionáveis, tais como astrologia, telepatia, simpatias e superstições comuns. Já o estilo de pensamento denominado de otimismo ingênuo indica o quanto as pessoas são demasiada e ingenuamente otimistas. Por fim, o estilo de pensamento negativo, está relacionado a comportamentos não-adaptativos (Saidel, Tróccoli \& Zannon, 2001).

Em geral, o pensamento construtivo está correlacionado positivamente com auto-estima, força do ego, bem-estar. As pessoas com elevados escores no Inventário de Pensamento Construtivo (CTI- Constructive Thinking Inventory) tendem a ser bem ajustadas (não-neuróticas), extrovertidas, cordiais, escrupulosas e de mente aberta. De modo contrário, pessoas que pontuam baixo tendem a ser neuróticas, introvertidas, hostis, inescrupulosas e de mentalidade estreita.

Ao contrário do pensamento construtivo, vinculado a fatores disposicionais do indivíduo, as estratégias de coping estão vinculadas a fatores situacionais, como, por exemplo, a expectativa de inserção ocupacional. As estratégias de $c o-$ ping podem mudar de momento para momento, durante os estágios de uma situação estressante. Dada a variabilidade nas reações individuais, não parece viável tentar predizer respostas situacionais apenas a partir do pensamento construtivo de uma pessoa. Faz-se necessário utilizar uma medida de estratégias de coping para tentar cercar o fenômeno estudado em sua diversidade. As estratégias de coping dizem respeito a comportamentos ou pensamentos usados para lidar com um estressor específico em uma situação específica (Folkman, Lazarus, Dunkel-Schetter, DeLongis \& Gruen, 1986, conforme citado por Antoniazzi, Dell'Aglio \& Bandeira, 1998).

A estratégia de coping está definida de forma constitutiva, neste estudo, em cinco dimensões: (1) Esquiva - cognições 
e comportamentos de manter-se longe do estressor. Compreende respostas como procurar esquecer, evitar o problema, fugir da situação, ir para outro lugar, deixar o tempo passar e ocupar o tempo com outras atividades; (2) Coping direto - o indivíduo apresenta cognições ou comportamentos que visam eliminar ou modificar o evento estressante. Nesse caso tratase de planejar uma solução e buscar informações de como se inserir no mercado de trabalho; (3) Apoio social - procura de apoio instrumental, emocional ou de informações com outras pessoas, de como se inserir no mercado de trabalho; (4) Emoções negativas - constituem-se de emoções e reações negativas, como por exemplo, tensão, falta de conhecimento de como buscar emprego, sentimento de incapacidade, dor de cabeça, sentir-se deprimido e auto-culpa; (5) Apego à religião - representa pensamentos e comportamentos religiosos que possam auxiliar no enfrentamento do problema.

\section{Saúde Mental e Inserção Ocupacional}

Evidências empíricas demonstram que os transtornos mentais comuns estão associados a variáveis relacionadas a condições de vida e à estrutura ocupacional. No primeiro caso, encontram-se a escolaridade, a posse de bens duráveis, de casa, de carro e condições de moradia. No segundo, podese citar a renda, a ocupação e a exclusão do mercado formal de trabalho (Ludermir \& Melo Filho, 2002).

De acordo com Kleinmand e Cohen (1997), recopilando dados da Organização Mundial de Saúde (OMS), nos últimos anos tem-se registrado um aumento substancial de pessoas que se queixam de sintomas que podem ser caracterizados como típicos de doenças mentais, entre as quais a depressão. Os transtornos mentais comuns caracterizam-se por sintomas como: insônia, fadiga, irritabilidade, esquecimento, dificuldade de concentração e queixas somáticas. De acordo com Ludermir e Melo Filho (2002), em países industrializados sua prevalência varia de $7 \%$ a $30 \%$.

No Brasil, estudos já foram realizados focalizando a transição dos jovens da escola/universidade para o mercado de trabalho. Por exemplo, Sarriera, Schwarez e Câmara (1996b) verificaram que o QSG12 (Questionário de Saúde Geral) é uma medida importante para a compreensão dos aspectos envolvidos nos estados de saúde e bem-estar psicológico do jovem, durante o período de transição escola-trabalho.

De modo geral, as pesquisas apontam a importância do coping para a promoção de comportamentos ajustados e são muitos os estudos que tratam de como as pessoas lidam com dificuldades ou circunstâncias estressantes em suas vidas e a implicação desses eventos na saúde mental e física (Aspinwall \& Taylor, 1997; Brissette \& cols., 2002; Carver \& Scheier, 1994; Epstein, 1992; Epstein \& Katz, 1992; Epstein \& Meier, 1989; Greenberger \& McLaughlin, 1998; Katz \& Epstein, 1991; Saidel \& cols., 2001). Entretanto, a maioria desses estudos trata dos estressores de forma muito abrangente e refere-se a amostras de estrangeiros.

No presente estudo, buscou-se desenvolver uma hipótese teórica de coping específica da expectativa e da situação de inserção ocupacional. Considerou-se a variável escolaridade como determinante da situação de transição. Os estudantes do terceiro ano do ensino médio e os universitários se inserem numa situação de transição da escola/universidade para o mercado de trabalho. As hipóteses aqui propostas, e descritas a seguir, apontam o pensamento construtivo e as estratégias de coping como preditores da expectativa de inserção ocupacional e de depressão. De acordo com a primeira hipótese, o indicador de depressão é influenciado pelos recursos de $c o$ ping, com os quais os jovens contam. O coping é usualmente considerado como sendo o mediador entre saúde mental e física (Hudek-Knežecić \& Kardum, 2000; Ryan-Wenger, 1992). Desse modo, o indicador de depressão é influenciado pelo pensamento construtivo e pelas estratégias de coping. E, conforme a segunda hipótese, a expectativa de inserção ocupacional é influenciada pelos recursos de coping, com os quais os jovens contam. Supõe-se que, além de ser um preditor do indicador de depressão, o pensamento construtivo e as estratégias de coping também podem predizer a expectativa de inserção ocupacional. Pode-se considerar, por exemplo, que os estudantes que apresentam em maior grau o pensamento construtivo e a estratégia de coping direto, apresentam também maior expectativa de inserção ocupacional.

\section{Método}

Trata-se de um estudo transversal, em que é utilizada uma metodologia uni, bi e multivariada de controle e análise de variáveis. As variáveis antecedentes, pensamento construtivo e estratégias de coping, são aqui entendidas como preditoras das variáveis conseqüentes - indicadores de depressão e expectativa de inserção ocupacional.

\section{Participantes}

Participaram deste estudo 413 estudantes, sendo 33,4\% do terceiro ano do ensino médio e $24,2 \%$ do ensino superior da cidade de Areia; $16,5 \%$ do terceiro ano do ensino médio e $25,9 \%$ do ensino superior da cidade de João Pessoa. Os participantes tinham, em média, 19 anos (DP = 2,74; mínimo $=15$ e máximo $=30)$ e a maioria deles era do sexo feminino $(55,1 \%)$.

\section{Procedimento}

Os instrumentos foram aplicados em escolas de ensino médio e universidades em Areia e João Pessoa. Utilizou-se um procedimento padrão, em que os aplicadores foram previamente treinados e orientados para intervir o mínimo possível nas respostas dadas pelos participantes, minimizando, assim, a possibilidade de viés nas respostas. Os questionários foram aplicados de forma coletiva, em salas de aula. Em um primeiro momento, foram passados para os participantes os esclarecimentos sobre o anonimato e sigilo, Obtido o consentimento verbal, os participantes foram orientados quanto ao preenchimento dos questionários. Foi garantido o caráter voluntário da participação, bem como o respeito às diretrizes éticas que regem a pesquisa com seres humanos.

\section{Instrumentos}

Para a coleta de dados, foi aplicado um questionário constituído por questões de levantamento socioeconômico e por quatro escalas de medida, a saber: 
- O pensamento construtivo foi mensurado por meio do Inventário de Pensamento Construtivo (Epstein \& Meier, 1989), em sua versão completa, em que os itens foram associados a uma escala Likert de cinco pontos de concordância. O inventário é formado por seis subescalas: coping emocional $(\alpha=0,85)$, coping comportamental $(\alpha=0,84)$, pensamento categórico $(\alpha=0,70)$, pensamento supersticioso $(\alpha=0,75)$, otimismo ingênuo $(\alpha=0,67)$ e pensamento negativo $(\alpha=$ $0,73)$.

- A estratégia de coping, específica da expectativa e da situação de inserção ocupacional, foi mensurada por meio de um questionário que mede as seguintes subescalas: emoções negativas $(\alpha=0,81)$, apoio social $(\alpha=0,78)$, esquiva $(\alpha=0,71)$, coping direto $(\alpha=0,67)$ e apego à religião $(\alpha=$ $0,69)$. Esse questionário foi construído a partir de medidas de estratégias de coping já existentes e de estudos qualitativos (Coelho, 2005). Os itens foram associados a uma escala de resposta Likert, de cinco pontos de concordância.

- Para aferir o indicador de depressão, utilizou-se o Questionário de Saúde Geral (QSG12). Este questionário corresponde a uma versão abreviada do Questionário de Saúde Geral, de Goldberg (1972), adaptado para o contexto brasileiro por Pasquali, Gouveia, Andriola, Miranda e Ramos (1994). Os itens utilizados para medir depressão foram: "Tem pensado que você é uma pessoa que não serve para nada?", "Tem perdido confiança em si mesmo(a)?", "Tem se sentido pouco feliz e deprimido(a)?", "Sente-se razoavelmente feliz considerando todas as circunstâncias?" e "Tem tido a sensação de que não pode superar suas dificuldades?".

- Para verificar a expectativa de inserção ocupacional, foram realizadas duas perguntas. A primeira diz respeito à profissão que os estudantes desejam exercer ("Qual a profissão que você gostaria de exercer?"). Foi solicitado que indicassem a possibilidade percebida de exercer a profissão escolhida, por meio de uma escala de sete pontos do tipo Likert $(1=$ Nenhuma possibilidade, $7=$ Todas as possibilidades). A segunda questão trata das possibilidades de inserção ocupacional que os jovens pensavam existir ("Quais as possibilidades de trabalho que você encontra hoje? Cite três."). Foi solicitado aos estudantes que citassem, no máximo, três opções. Para este estudo, interessou apenas o número de possibilidades apresentado. As respostas obtidas nessas duas questões foram somadas e o resultado dividido por dois, constituindo o índice de expectativa de exercer a profissão desejada e de inserção ocupacional.

\section{Análise dos dados}

Inicialmente, por meio do software SPSS (Statistical Package for the Social Sciences, em sua versão 15.0), foi detectado que menos de $5 \%$ dos dados das auto-avaliações foram deixados em branco pelos respondentes. Optou-se por substituí-los pela média geral. Verificou-se, nos gráficos de dispersão, a existência de multicolinearidade entre algumas variáveis preditoras. Essas relações poderiam levar a problemas nos modelos de regressão. A solução para esses problemas será apresentada nas seções de Resultados e Discussão.

Para a validade de construto, primeiro verificaram-se os índices de Kaiser-Meyer-Olkin e o teste de Esfericidade de
Bartlett - medidas que verificam a fatoriabilidade da matriz de correlação entre os itens da escala, para serem então submetidos à análise fatorial exploratória. Essa última análise tem como objetivo verificar a dimensionalidade dos instrumentos de medida. Além disso, os dados foram submetidos à análise da técnica alfa de Cronbach para verificar a precisão da medida (Pasquali, 1997, 2003).

Para identificar a estrutura empírica das medidas para essa amostra, todas as escalas foram submetidas à análise fatorial exploratória. Foram realizadas análises de correlação $r$ de Pearson e Regressão Linear Múltipla (hierárquica), para verificar o nível de relação e em que medida as variáveis antecedentes explicam as variáveis conseqüentes, também com o SPSS. Bem como uma equação por modelagem estrutural para testar o modelo teórico por meio do software AMOS 6.0.

Resumem-se, a seguir, alguns dos principais indicadores de qualidade de ajuste da análise do modelo:

- Qui-quadrado $\left(\chi^{2}\right)$. Comprova a probabilidade do modelo se ajustar aos dados. Um valor do $\chi^{2}$ estatisticamente significativo indica discrepâncias entre os dados e o modelo teórico que está sendo testado. É influenciado pelo tamanho da amostra e assume a multinormalidade do conjunto de variáveis.

- Índice de Qualidade do Ajuste (Goodness-of-Fit Index, $G F I$ ) e o Índice de Qualidade do Ajuste Ajustado (Adjusted Goodness-of-Fit Index, $A G F I$ ), que é ponderado em função dos graus de liberdade do modelo, com respeito ao número de variáveis consideradas. É recomendado valores do GFI e AGFI superiores ou próximos a 0,95 e 0,90 , respectivamente (Garson, 2003; Hu \& Bentler, 1999). Estas estatísticas não são influenciadas pelo tamanho da amostra dos participantes (Saris \& Stronkhorst, 1984).

- Razão $\chi^{2} /$ gl. É considerada uma qualidade de ajuste subjetiva (Byrne, 1989). Um valor inferior a 5,0 pode ser interpretado como indicador da adequação do modelo teórico para descrever os dados.

- Raíz Quadrada Média Residual (Root Mean Square Residual, RMR). Baseia-se nos residuais, sendo que um valor próximo a zero significa que o modelo se ajusta aos dados, indicando que todos os residuais se apresentam mais perto desse valor. Considerar-se-á a RMR padronizada em que um valor em torno de 0,05 é considerado uma prova da adequação do modelo (Saris \& Stronkhorst, 1984).

- Raíz quadrada média do erro de aproximação (Root Mean Square Error of Approximation, RMSEA). Com relação aos valores de RMSEA, recomendam-se valores próximos a 0,06 e 0,08, respectivamente (Hu \& Bentler, 1999).

\section{Resultados e Discussão}

\section{Aspectos psicométricos}

A análise fatorial exploratória, com método dos Componentes Principais e rotação Varimax, indicou que o Inventário de Pensamento Construtivo, proposto por Epstein e Méier (1989), replicou a estrutura fatorial, com cargas fatoriais variando entre 0,30 e 0,72 . Igualmente, a escala de estratégia de coping, específica da expectativa e da situação de inserção ocupacional, apresentou rigorosamente a estrutura teórica 
estabelecida a priori, com cargas fatoriais variando entre 0,38 e 0,69. O Questionário de Saúde Geral - QSG12, por meio de uma rotação Direct Oblimin, apresentou uma estrutura tri-fatorial, comum aos estudos realizados com amostra de brasileiros (Sarriera, Schwarcz \& Câmara, 1996b), com cargas fatoriais variando entre 0,52 e 0,78 .

\section{Coping como preditor do indicador de depressão e da expectativa de inserção ocupacional}

Com a finalidade de testar a predição das subescalas coping comportamental e emocional, do pensamento construtivo e as estratégias de coping em relação ao indicador de depressão e à expectativa de inserção ocupacional, realizouse uma análise de Regressão Linear Múltipla (hierárquica). Optou-se por considerar apenas as subescalas coping emocional e comportamental, do pensamento construtivo, por seus conteúdos representarem o pensamento construtivo propriamente dito, por meio do pensamento producente. Embora a dimensão pensamento negativo apresentasse bom índice de consistência interna, esta foi desconsiderada, para eliminar o efeito de multicolinearidade com a dimensão emoções negativas, da escala de estratégias de coping e para evitar tautologias. Os resultados dessa análise são apresentados nas Tabelas 1 e 2 .

De acordo com os resultados apresentados na Tabela 1, os melhores preditores para a dimensão depressão foram: coping emocional $(\beta=-0,22)$, coping comportamental $(\beta=0,12)$, emoções negativas $(\beta=0,18)$, esquiva $(\beta=0,11)$ e coping direto $(\beta=0,12)$, com explicação de $21 \%$ da variabilidade de depressão $\left(\mathrm{R}^{2}=0,21\right)$. Quanto mais as pessoas apresentaram coping emocional, coping comportamental e coping direto, menos apresentaram os indicadores de depressão. Já as pessoas que apresentaram mais fortemente emoções negativas e esquiva tenderam a apresentar em maior grau os indicadores de depressão.

Tratando da relação entre os construtos, verificou-se que o pensamento negativo está positivamente correlacionado com emoções negativas, com apoio social, com esquiva e com apego à religião, enquanto correlaciona-se negativamente com coping direto. Esses resultados eram esperados, pois se supõe que a dimensão pensamento negativo, principalmente, deveria estar relacionada positivamente com emoções negativas e esquiva, e negativamente com coping direto. A dimensão coping emocional correlacionou-se negativamente com emoções negativas, esquiva e apego à religião. $\mathrm{O}$ coping comportamental está correlacionado negativamente com emoções negativas e esquiva e positivamente com apoio social e coping direto. Pode-se supor que as relações existentes entre essas variáveis são consistentes com a definição operacional de cada uma delas e que, de certo modo, esses resultados indicam a possibilidade de validade convergentediscriminante da escala de estratégia de coping, frente à expectativa de inserção ocupacional.

Os participantes com pontuação mais alta nas dimensões coping emocional e comportamental tendem a apresentar menores índices de depressão. As pessoas que apresentam em maior grau coping emocional são capazes de enfrentar situações difíceis sem sofrer estresse em demasia. As pessoas com elevados índices de coping comportamental são entusiásticas, otimistas, enérgicas, extrovertidas e escrupulosas, tendem a agir elaborando previamente um plano estratégico.

As evidências empíricas demonstram que emoções negativas e esquiva estão correlacionadas positivamente com

Tabela 1. Pensamento construtivo e estratégias de coping como preditores do indicador de depressão.

\begin{tabular}{|c|c|c|c|c|c|c|c|c|c|c|c|}
\hline & & & & & & & & & \multicolumn{3}{|c|}{ Depressão } \\
\hline & De & $\mathrm{Ce}$ & $\mathrm{Cc}$ & Em & As & Es & Cd & Ar & B & $\beta$ & $t$ \\
\hline $\mathrm{Ce}$ & $-0,31 * *$ & & & & & & & & $-0,19$ & $-0,22$ & $-4,76^{* *}$ \\
\hline $\mathrm{Cc}$ & $-0,22 * *$ & $0,21 * *$ & & & & & & & $-0,12$ & $-0,12$ & $-2,38^{* *}$ \\
\hline Em & $0,31 * *$ & $-0,18^{* *}$ & $-0,12 *$ & & & & & & 0,13 & 0,18 & $3,64 * *$ \\
\hline As & 0,04 & 0,06 & $0,30 * *$ & $0,22 * *$ & & & & & 0,06 & 0,09 & 1,67 \\
\hline Es & $0,25 * *$ & $-0,15^{*}$ & $-0,27 * *$ & $0,30 * *$ & $-0,01$ & & & & 0,10 & 0,11 & $2,30 *$ \\
\hline $\mathrm{Cd}$ & $-0,19 * *$ & $0,12 *$ & $0,32 * *$ & $-0,15^{*}$ & $0,37 * *$ & $-0,22 * *$ & & & $-0,09$ & $-0,12$ & $-2,36^{*}$ \\
\hline $\mathrm{Ar}$ & 0,08 & $-0,12 *$ & 0,07 & $0,12 *$ & $0,28 * *$ & 0,00 & $0,30 * *$ & & 0,03 & 0,05 & 1,13 \\
\hline Média & 1,69 & 3,18 & 3,99 & 2,99 & 3,46 & 1,61 & 3,49 & 3,16 & & & \\
\hline Desvio padrão & 0,59 & 0,69 & 0,57 & 0,83 & 0,81 & 0,62 & 0,80 & 0,86 & & & \\
\hline & & & & & & & & & \multicolumn{3}{|c|}{ Intercept $=2,22$} \\
\hline & & & & & & & & & \multicolumn{3}{|c|}{$r=0,46$} \\
\hline & & & & & & & & & \multicolumn{3}{|c|}{$\mathrm{R}^{2}=0,21$} \\
\hline & & & & & & & & & \multicolumn{3}{|c|}{$\mathrm{R}^{2}$ Ajus $=0,20$} \\
\hline & & & & & & & & & \multicolumn{3}{|c|}{$\mathrm{F}(7,412)=15,81 * *$} \\
\hline
\end{tabular}

Nota. ${ }^{*} \mathrm{p}<0,05, *{ }^{*} \mathrm{p}<0,01$. B (Unstandardized Coefficients) $=$ inclinação da reta de regressão; $\beta$ (Standardized Coefficients) $=$ indicador da relação entre VD e VI's; $t=$ teste $\mathrm{t} ; \mathrm{R}^{2}=$ proporção de variância explicada; Razão $\mathrm{F}=$ probabilidade associada ao $\mathrm{R}^{2}$. De $=$ depressão, Ce $=$ coping emocional, Cc $=$ coping comportamental, $\mathrm{Em}=$ emoções negativas, $\mathrm{As}=$ apoio social, $\mathrm{Es}=$ esquiva, $\mathrm{Cd}=$ coping direto e Ar $=$ apego à religião. $\mathrm{O}$ valor estatisticamente significante do Beta é apresentado em negrito. 
Tabela 2. Pensamento construtivo e estratégias de coping como preditores da expectativa de exercer a profissão desejada e de inserção ocupacional.

\begin{tabular}{|c|c|c|c|c|}
\hline & \multirow{2}{*}{ Expectativa } & \multicolumn{3}{|c|}{ Expectativa } \\
\hline & & $\mathbf{B}$ & $\boldsymbol{\beta}$ & $t$ \\
\hline Coping emocional & $-0,00$ & $-0,11$ & $-0,07$ & $-1,41$ \\
\hline Coping comportamental & $0,12 * *$ & 0,14 & 0,07 & 1,35 \\
\hline Emoções negativas & $-0,18 * *$ & $-0,19$ & $-0,14$ & $-2,74 * *$ \\
\hline Apoio social & 0,00 & $-0,02$ & $-0,02$ & $-0,35$ \\
\hline Esquiva & $-0,12 * *$ & $-0,08$ & $-0,04$ & $-0,86$ \\
\hline Coping direto & $0,15^{* *}$ & 0,17 & 0,12 & $2,25^{*}$ \\
\hline Apego à religião & $-0,01$ & $-0,05$ & $-0,04$ & $-0,85$ \\
\hline Média & 3,94 & & & \\
\hline \multirow[t]{6}{*}{ Desvio padrão } & 1,09 & & & \\
\hline & & \multicolumn{3}{|c|}{ Intercept $=4,11$} \\
\hline & & \multicolumn{3}{|l|}{$r=0,25$} \\
\hline & & \multicolumn{3}{|c|}{$\mathrm{R}^{2}=0,06$} \\
\hline & & \multicolumn{3}{|c|}{$\mathrm{R}^{2}$ Ajus. $=0,04$} \\
\hline & & \multicolumn{3}{|c|}{$\mathrm{F}(7,412)=3,92 * *$} \\
\hline
\end{tabular}

a depressão, enquanto o coping direto está negativamente correlacionado com depressão. Esses resultados eram esperados, pois as emoções negativas dizem respeito a reações negativas, tensão, falta de conhecimento de como buscar emprego, sentimento de incapacidade, dor de cabeça, sentirse deprimido e auto-culpa, entre outros. Esquiva é constituída por cognições e comportamentos de manter-se longe do fator estressor. Já o coping direto - cognições ou comportamentos que visam eliminar ou modificar o evento estressante - parece mais distante dos conteúdos dos indicadores de depressão: irritabilidade, insônia e sentimento de incapacidade.

A predição para a expectativa de exercer a profissão desejada e de inserção ocupacional foi providenciada por emoções negativas $(\beta=-0,14)$ e coping direto $(\beta=0,12)$, com explicação de $6 \%$ da variabilidade $\left(\mathrm{R}^{2}=0,06\right)$. Embora a explicação providenciada não seja ideal, é meritória. E, nesse sentido, os resultados corroboram os pressupostos teóricos, pois quanto mais coping direto o indivíduo apresentar, maior o índice de expectativa, enquanto que apresentando em maior grau emoções negativas, tem-se menor índice de expectativa (ver Tabela 2).

Para testar o modelo teórico proposto, foi realizada uma modelagem por equações estruturais. No modelo apresentado na Figura 1, o pensamento construtivo, constituído por seus dois fatores, coping emocional e comportamental, está como explicador da variável estratégias de coping, representada pelas dimensões coping direto, emoções negativas e esquiva que, por sua vez, explicam os indicadores de depressão e de expectativa.

A estimativa dos parâmetros foi realizada por meio do ML (Maximum Likelihood). Os índices de ajuste do modelo aos dados foram bastante satisfatórios $\left(\chi^{2} / \mathrm{gl}=2,31\right.$; AGFI = $0,94$; GFI $=0,97 ; \mathrm{RMR}=0,03$; RMSEA $=0,05)$. Esses indicadores possibilitam afirmar que o modelo proposto se ajusta aos dados da amostra estudada. Desse modo, corrobora a hipótese de que o pensamento construtivo é explicador das estratégias de coping $(\beta=0,90)$ que, por sua vez, explicam os indicadores de depressão $(\beta=-0,57)$ e a expectativa $(\beta=0,28)$. Também corrobora a hipótese de que o pensamento construtivo, composto pelas dimensões coping comportamental e emocional, é explicador das estratégias de coping, constituídas pelas dimensões coping direto, esquiva e emoções negativas que explicam os indicadores de depressão e de expectativa.

\section{Considerações Finais}

É importante recuperar aqui o objetivo principal deste estudo: objetivou-se estudar o pensamento construtivo e as estratégias de coping, tanto como explicadores das expectativas de inserção ocupacional dos jovens, quanto como indicadores de depressão.

Verificou-se que os jovens que utilizam recursos de coping, do tipo coping comportamental e emocional (dimensões do pensamento construtivo), e coping direto (dimensão das estratégias de coping), são os que melhor enfrentam as pressões da transição da escola/universidade para o mercado de trabalho, com menores níveis de depressão e melhores expectativas.

Com esses resultados, espera-se fornecer informações que ampliem o corpo de conhecimento da Psicologia Social, possibilitando, além disso, instrumentalizar melhor as políticas públicas voltadas para os jovens. Espera-se, também, 


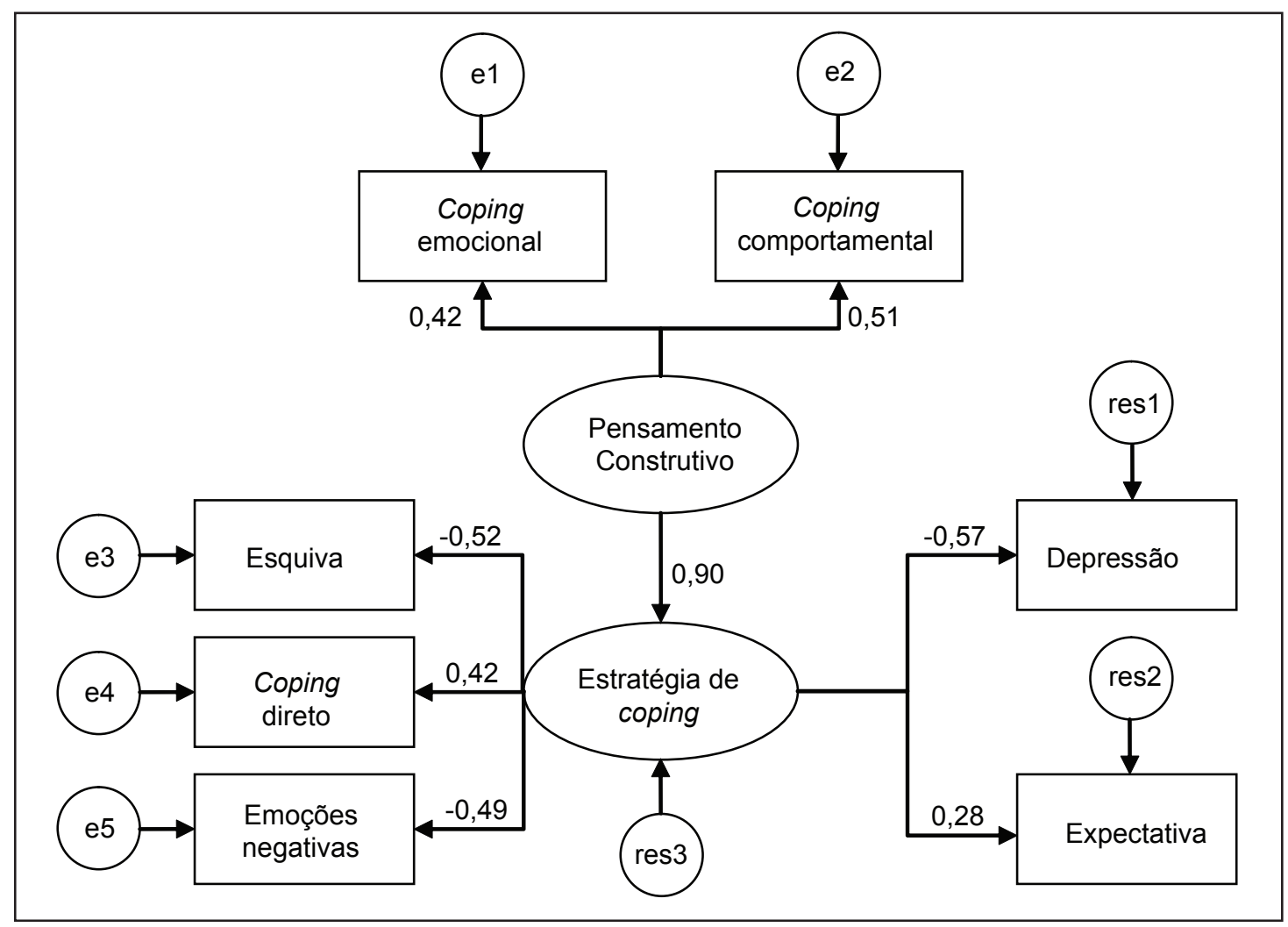

Figura 1. Modelo explicativo dos indicadores de depressão e da expectativa de exercer a profissão desejada/inserção ocupacional a partir do pensamento construtivo e das estratégias de coping (e1, e2, e3, e4 e e5 = parâmetro de erro; res1, res2 e res3 = erro residual).

contribuir para incentivar trabalhos de intervenção, que auxiliem os jovens na busca de sua primeira ocupação, por meio de agentes de órgãos governamentais e não-governamentais. Finalmente, busca-se fomentar conhecimentos para o atendimento clínico, principalmente nas terapias comportamentais, o que facilitará aos jovens identificar os estilos e as estratégias de coping que menos geram efeitos psicológicos negativos para eles.

No âmbito clínico, um primeiro passo é estabelecer normas diagnósticas. Para tanto, novas amostras devem ser constituídas, considerando uma maior diversidade de participantes. A partir de então, será possível orientar e intervir, de modo a privilegiar um tipo específico de coping, que possa trazer mais benefícios para as pessoas.

Uma das limitações deste estudo diz respeito ao corte transversal, que chama atenção para o tempo, como uma importante variável a ser levada em conta. No tocante à amostragem, seria proveitoso ampliar a coleta de dados, utilizando jovens sem nenhuma ocupação institucional. Ponderando-se essas limitações, pode-se dizer que o estudo cumpriu o objetivo principal a que se propôs, verificando as relações entre o pensamento construtivo, estratégias de coping, depressão e expectativa.

É importante salientar o papel do psicólogo social na promoção de comportamentos ajustados e na avaliação de programas, como, por exemplo, o do primeiro emprego, implementado pelo Governo Federal. A eficácia desse tipo de iniciativa depende, fundamentalmente, de uma avaliação de seu impacto, em termos de profundidade e de amplitude.
O psicólogo social é capaz de realizar trabalhos de avaliação de políticas públicas, na área das organizações, sobre cooperativismo e associacionismo, resolução de conflitos, transferência de tecnologias, saúde mental e bem-estar psicológico (Albuquerque, 2002).

Por fim, o estudo tornou evidente que os jovens que utilizam recursos de coping (como o coping comportamental e o coping emocional) e estratégias de coping (como o coping direto) são os que melhor enfrentam as pressões da transição da escola/universidade para o mercado de trabalho, com menores níveis de depressão e melhores expectativas.

\section{Referências}

Albuquerque, F. J. B. (2002). Psicologia social e formas de vida rural no Brasil. Psicologia: Teoria e Pesquisa, 18, 37 - 42.

Antoniazzi, A. S., Dell'Aglio, D. D. \& Bandeira, D. R. (1998). A evolução do conceito de coping: Uma revisão teórica. Estudos de Psicologia, 3, 273-294.

Aspinwall, L. G. \& Taylor, S. E. (1997). A stitch in time: Selfregulation and proactive coping. Psychological Bulletin, 121, 417-436.

Brissette, I., Scheier, M. F. \& Caver, C. S, (2002). The role of optimism in social network development, coping, and psychological adjustment during a life transition. Journal of Personality and Social Psychology, 82, 102-111.

Byrne, B.M. (1989). A primer of LISREL: Basic applications and programming for confirmatory factor analytic models. New York: Springer-Verlag. 
Caver, C. S. \& Scheir, M. F. (1994). Situational coping and coping dispositions in a stresseful transaction. Journal of Personality and Social Psychology, 66, 184-195.

Coelho, J. A. P. M. (2005). Coping, ansiedade e depressão em jovens do ambiente urbano e rural frente à expectativa de inserção ocupacional. Dissertação de Mestrado, Universidade Federal da Paraíba, João Pessoa.

Criterium Assessoria (2004). Perfil da juventude brasileira. Retirado em 10/09/2004 de http://www.sebrae.com.br/br/download/ perfil_juventude_brasileira.pps

Epstein, S. (1992). Coping ability, negative self-evaluation, and overgeneralization: Experiment and theory. Journal of Personality and Social Psychology, 62, 826-836.

Epstein, S. \& Katz, L. (1992). Coping ability, stress, productive load, and symptoms. Journal of Personality and Social Psychology, 62, 813-825.

Epstein, S. \& Meier, P. (1989). Constructive thinking: A broad coping variable with specific components. Journal of Personality and Social Psychology, 57, 332-350.

Garson, G. D. (2003). PA 765 Statnotes: An online textbook. Retirado em 24/11/2004 de http://www2.chass.ncsu.edu/garson/ pa765/statnote.htm

Goldberg, D. P. (1972). The detection of psychiatric illness by questionnaire. Londres: Oxford University Press.

Greenberger, E. \& McLaughlin, C. S. (1998). Attachament, coping, and explanatory style in late adolescence. Journal of Youth and Adolescence, 27, 121-139.

Hu, L.-t., \& Bentler, P. M. (1999). Cutoff criteria for fit indexes in covariance structure analysis: Conventional criteria versus new alternatives. Structural Equation Modeling, 6, 1-55.

Hudek-Knežecić, J. \& Kardum, I. (1996). A model of coping with conflicts between occupational and family roles: structural analysis. Personality and Individual Differences, 21, 355372.

Hudek-Knežecić, J. \& Kardum, I. (2000). The effects of dispositional and situational coping, perceived social support, and cognitive appraisal on immediate outcome. European Journal of Psychological Assessment, 16, 190-201.

Katz, L. \& Epstein, S. (1991). Constructive thinking and coping with laboratory-induced stress. Journal of Personality and Social Psychology, 61, 789-800.

Kleinmand, A. \& Cohen, A. (1997). Psychiatry's global challenge. Scientific American, 276, 86-89.

Ludermir, A. B. \& Melo Filho, D. A. (2002). Condição de vida e estrutura ocupacional associados a transtornos mentais comuns. Revista Saúde Pública, 36, 213-221.
Luque, A. G. \& Estramiana, J. L. Á. (2000). La investigación psicológica sobre el desempleo juvenil. Em E. A. Tomás, C. Remeseiro \& J. A. F. Alonso (Orgs.), Psicologia del trabajo de lãs organizaciones y de los recursos humanos (pp. 211-215). Madrid: Biblioteca Nueva.

Myers, D. (1998). Psicologia social. Rio de Janeiro: LTC.

Organização Internacional do Trabalho (2001). Desemprego juvenil no Brasil. Brasília: OIT

Pasquali, L. (1997). Psicometria: teoria e aplicações. Brasília: Universidade de Brasília.

Pasquali, L. (2003). Psicometria: teoria dos testes na psicologia e na educação. Petrópolis: Vozes.

Pasquali, L., Gouveia, V.V., Andriola, W.B., Miranda, F.J. \& Ramos, A.L.M. (1994). Questionário de Saúde Geral de Goldberg (QSG): adaptação brasileira. Psicologia: Teoria e Pesquisa, 10, 421-437.

Ryan-Wenger, N. M. (1992). A taxonomy of children's coping strategies: A step toward theory development. American Journal of Orthopsychiatry, 62, 256-263.

Saidel, E. M. F., Tróccoli, B. T. \& Zannon, C. M. L. C. (2001). Análise fatorial de uma medida de estratégia de enfrentamento. Psicologia: Teoria e Pesquisa, 17, 225-234.

Saris, W. \& Stronkhorst, H. (1984). Causal modeling in nonexperimental research. Amsterdam: Sociometric Research Foundation.

Sarriera, J. C., Schwarez, C. \& Câmara, S. G. (1996a). Juventude, ocupação e saúde. Coletâneas da ANPEPP: Aplicações da Psicologia na melhoria da qualidade de vida, 1, 61-78.

Sarriera, J. C., Schwarez, C. \& Câmara, S. G. (1996b). Bem-estar psicológico: análise fatorial da escala de Goldberg (GHQ-12) numa amostra de jovens. Psicologia: Reflexão e Crítica, 9, 293-306.

Smith, E. R. \& Mackie, D. M. (1997). Psicología social. Madrid: Panamericana. 